\title{
Development of the REFOCUS intervention to increase mental health team support for personal recovery
}

Mike Slade, Victoria Bird, Clair Le Boutillier, Marianne Farkas, Barbara Grey, John Larsen, Mary Leamy, Lindsay Oades and Julie Williams

\section{Background}

There is an emerging evidence base about best practice in supporting recovery. This is usually framed in relation to general principles, and specific pro-recovery interventions are lacking.

\section{Aims}

To develop a theoretically based and empirically defensible new pro-recovery manualised intervention - called the REFOCUS intervention.

\section{Method}

Seven systematic and two narrative reviews were undertaken. Identified evidence gaps were addressed in three qualitative studies. The findings were synthesised to produce the REFOCUS intervention, manual and model.

\section{Results}

The REFOCUS intervention comprises two components: recovery-promoting relationships and working practices. Approaches to supporting relationships comprise coaching skills training for staff, developing a shared team understanding of recovery, exploring staff values, a Partnership Project with people who use the service and raising patient expectations. Working practices comprise the following: understanding values and treatment preferences; assessing strengths; and supporting goal-striving. The REFOCUS model describes the causal pathway from the REFOCUS intervention to improved recovery.

\section{Conclusions}

The REFOCUS intervention is an empirically supported prorecovery intervention for use in mental health services. It will be evaluated in a multisite cluster randomised controlled trial (ISRCTN02507940).

\section{Declaration of interest}

None.

\section{Copyright and usage}

(c) The Royal College of Psychiatrists 2015.
National mental health policy mandates a recovery orientation in many countries. Implementing this policy vision in mental health systems is challenging. ${ }^{1}$ The National Institute of Health Research funded the REFOCUS programme between 2009 and 2014 to support the development of a recovery orientation in adult mental health services. The programme of work was undertaken in two phases. In phase 1, existing evidence was synthesised through a series of systematic and comprehensive reviews, and new primary research was undertaken. The deliverables from phase 1 were a new manualised intervention, called the REFOCUS intervention, ${ }^{2}$ including a testable description of the causal pathway between intervention and improved recovery, called the REFOCUS model. The intervention and model were tested in phase 2. The aim of this paper is to describe the phase 1 work.

\section{Method}

\section{Design}

The scientific framework for the REFOCUS programme was the MRC Framework for Complex Interventions, ${ }^{3}$ which proposes that complex interventions be developed from the systematic use of a clear theoretical basis. Phase 1 reported here involved three stages: (a) synthesis of theory to identify overarching principles, (b) development and manualisation of the REFOCUS intervention and (c) development of the testable REFOCUS model. The intervention built on existing research, synthesised either using systematic or narrative reviews (specifically 'systematised' reviews ${ }^{4}$ which use some but not all features of a systematic review). Qualitative studies using interviews and focus groups addressed identified knowledge gaps. Ethical approval was obtained (South London REC Office (2) 10/H0807/4).

\section{Setting}

Multidisciplinary community mental health teams, providing case management primarily through patient (typically aged 18-65)/ worker meetings, and often involving long-term rather than episodic care.

\section{Procedure}

\section{Stage 1: theory}

Three underpinning principles were predefined. First, meaningful involvement from people with lived experience in the REFOCUS programme was prioritised, in acknowledgement of the concern expressed by some that the patient-developed notion of recovery can be seen to be "hijacked" ${ }^{5}$ by services and incorporated into the language of the mental health system without any substantive change to practice. Second, there are known inequalities in the experience of patients from minority ethnic groups. The REFOCUS programme therefore placed a particular emphasis on supporting recovery for Black people, who in England are a minority ethnic group with high psychosis prevalence and problematic pathways to care. Third, the intervention was intended to place less emphasis on diagnosis as the determinant of care, and therefore was transdiagnostic. However, as one objective for the REFOCUS programme was to inform clinical guidelines, which are indexed on diagnosis, the evaluation of the intervention would be in relation to its impact on people with psychosis. 
Reviewing all pro-recovery interventions to identify those with strongest evidence was considered, but specificity proved an insurmountable review challenge. The term 'recovery' occurred too frequently in titles and abstracts to be a useable search term, and the individual nature of recovery allowed almost any intervention or outcome to be seen as a contributor. The need for a more coherent theory base for the construct of recovery was identified.

Published descriptions and models of personal recovery were analysed to develop a conceptual framework for personal recovery. ${ }^{6}$ A narrative review included 97 papers with 87 distinct models, from 5208 screened and 366 reviewed. Narrative synthesis was used to develop a conceptual framework. To investigate generalisability, the systematic review was updated (7431 screened, 429 reviewed, 105 included) with a new focus on the country of origin of included studies. ${ }^{7}$ Owing to the paucity of recovery research relating to minority ethnic groups, a qualitative study of Black people using mental health patients was undertaken involving four focus groups $(n=26)$ and 14 individual interviews in four mental health trusts and two voluntary sector organisation across England (Qualitative Study 1).

The conceptual framework is based on retrospective reports of people reflecting on their recovery, so it may not be applicable to current patients who may be at an earlier stage of recovery. To investigate the applicability of the conceptual framework to people currently using mental health services, a qualitative study involving seven focus groups with 48 current mental health patients was undertaken in three areas of England (Qualitative Study 2). ${ }^{8}$ Participants were asked about their understanding and experience of personal recovery, with responses analysed using a constant comparison approach to validate the conceptual framework (deductive analysis) and identify new themes (inductive analysis).

To provide an organising framework for locating the intervention, a narrative review was undertaken, involving a thematic analysis of 30 recovery guidelines from six countries. ${ }^{9}$ The emergent Recovery Practice Framework synthesised the findings from best practice resources internationally. Candidate interventions were evaluated for their feasibility using a new measure called Structured Assessment of FEasibility (SAFE)..$^{10,11}$

A specific knowledge gap was identified in relation to staff perspectives on contextual barriers to and enablers of recoveryoriented practice. Therefore, a grounded theory study was undertaken, involving 10 focus groups with multidisciplinary clinicians $(n=34)$ and team leaders $(n=31)$ from five mental health trusts across England, followed by individual interviews with clinicians $(n=18)$, team leaders $(n=6)$ and senior managers $(n=8$; Qualitative Study 3). ${ }^{12}$

Empirical evidence relating to candidate components of the intervention was reviewed. Consideration was given to undertaking systematic reviews for each element, but this was disproportionate in likely benefit. Therefore, systematic reviews relating to assessment of strengths, ${ }^{13}$ hope,${ }^{14}$ measuring recovery ${ }^{15}$ and measuring recovery orientation ${ }^{16}$ were completed, along with a narrative review on social influences on recovery. ${ }^{17}$

\section{Stage 2: REFOCUS intervention and manual}

On the basis of Stage 1, a proposed structure for the REFOCUS intervention was developed by the research team. Expert input was then obtained from five advisory groups: a Lived Experience Advisory Panel (LEAP) of patients and carers $(n=8)$; a steering group of topic-specific experts $(n=19)$; a virtual advisory panel of patients, researchers and other stakeholders with an interest in Black people and minority ethnic mental health $(n=10)$; an international advisory board of international experts $(n=8)$; and individual consultees $(n=11)$. A particular focus was on ensuring meaningful patient and public involvement, so the impact of the LEAP was evaluated in relation to input from other advisory committees, and shown to be influential on the study design and implementation. ${ }^{18}$ The five advisory groups were consulted on the proposed structure for the REFOCUS intervention, in relation to external validity (is it targeting recovery rather than some other aspect of good practice?), feasibility (for community mental health team implementation), level of ambition (the right level of change from current practice) and resources (specific intervention or tools).

A draft manual was then developed, based on the findings from Stage 1 and the advisory committee consultation on the proposed structure. The advisory committees were then consulted again on the draft manual, in relation to feasibility (time, resources, skills), clarity (comprehensible, clinical fit), presentation (language, concepts, layout) and applicability (overlap with current practice, appropriate level of behaviour change). The draft manual was modified based on responses to produce the final REFOCUS intervention and REFOCUS manual. ${ }^{2}$

\section{Stage 3: REFOCUS model}

Stages 1 and 2 were synthesised to develop the REFOCUS model, a description of the intervention, the proposed mediators, and the outcome. The intervention primarily focused on workers, and understanding of practice change was informed by the theory of planned behaviour. ${ }^{19}$ This theory proposes that behavioural intent is influenced by attitudes and subjective norms, and by the perceived level of behavioural control. Meta-analysis of health research suggests that the theory accounts for over $20 \%$ of actual behaviour. ${ }^{20}$

\section{Results}

\section{Stage 1 (Theory)}

The conceptual framework produced three findings. ${ }^{6}$ First, 13 characteristics emerged: recovery is an active process; individual and unique process; non-linear process; recovery as a journey; recovery as stages or phases; recovery as a struggle; multidimensional process; recovery is a gradual process; recovery as a life-changing experience; recovery is possible without cure; recovery is aided by supportive and healing environment; recovery can occur without professional intervention; and trial and error process. Second, five key recovery principles were evident in recovery narratives: Connectedness, Hope and optimism, Identity, Meaning and purpose, and Empowerment giving the CHIME framework. Finally, the review identified that recovery narratives are consistent with a stages model, in which the journey of recovery is a continuous and unfolding process rather than a discontinuous one-off experience.

The updated cross-cultural systematic review showed that most recovery literature comes from English-speaking countries, ${ }^{7}$ so caution is needed in generalising the recovery construct to non-majority populations. Thematic analysis of the experience of Black people in Qualitative Study 1 indicated the central importance of individualised care based on the person's values and treatment preferences, rather than a 'one-size-fits-all' approach to planning services.

The focus group study of current mental health patients (Qualitative Study 2) validated the conceptual framework, and identified three areas of greater emphasis: practical support; diagnosis and medication; and scepticism surrounding recovery. ${ }^{8}$

The recovery-oriented practice framework identified four practice domains of recovery support: promoting citizenship 
(e.g. challenging stigma), organisational commitment (e.g. workforce planning), supporting personally defined recovery (e.g. treatments) and working relationship (e.g. interpersonal style). ${ }^{9}$ Candidate interventions at the level of promoting citizenship (e.g. community links) and organisational commitment (e.g. peerrun services) were evaluated using SAFE, and deemed infeasible within available resources.

The grounded theory study of staff perspectives on barriers and enablers ${ }^{12}$ found that staff had a range of opinions about recovery-oriented practice, reflecting their need to balance competing priorities and demands placed on them. These studies all informed the principles underpinning the REFOCUS intervention, shown in Table 1.

\section{Stage 2 (REFOCUS intervention and manual)}

A draft structure for the REFOCUS intervention was developed, with interventions described in outline and organised to correspond with care processes of assessment, intervention and evaluation. The structure comprised four core intervention modules (Knowledge, Values and attitudes, Strengths assessment, and Recovery planning and Interpersonal style) and five optional modules (Connectedness, Hope, Identity, Meaning and Empowerment). Modules used familiar clinical terminology and the intervention comprised the four core plus one optional module.

Consultation with advisory committees on the draft structure produced 16 responses, identifying five main themes: feasibility, implementation, suggested interventions or resources, patient involvement, and language. Feasibility concerns included resources, time needed to implement the manual and the staff skill set. The manual included too many components, and the core and optional structure was overcomplicated and made analysis more difficult. The need was identified for implementation strategies, which identify specifically how the intervention is implemented. References for suggested interventions or resources were accessed and reviewed. Patient involvement spanned development of the manual (which should be visible and explicit), providing the intervention (staff training should involve people with lived experience). Respondents did not agree with the use of clinical language, suggesting instead that the language used should represent and be consistent with the concept of personal recovery: 'I think it could be a mistake to try and dress the recovery approach in clinical language, as in my experience people see through it and feel uncomfortable with it and we should not be making apologies for what we are trying to achieve'.

On the basis of the consultation, a full draft of the REFOCUS intervention was developed. Consultation with the advisory committees on the full draft produced 14 responses, with five emergent themes. The theme 'service user involvement' related to amplifying the role of patients in the intervention. Adopted suggestions included informing patients about the intervention, raising their expectations to expect recovery-oriented care, emphasising staff-patient relationships involving trust, partnership and mutual respect, and facilitating an experience for staff and patients of working together on a common goal (the Partnership Project, described later). The theme 'training practicalities' emerged from clinicians and researchers, and related to the cost, timing and back-fill arrangements for training. The theme 'language' related to ensuring pro-recovery language in the REFOCUS manual and the issue of including people with English as an additional language. The 'implementation' theme related to implementation of the intervention in clinical practice. Finally, many resources were suggested and reviewed.

The draft manual was modified to produce the final version of the REFOCUS manual. ${ }^{2}$ The manual provides resources to implement the REFOCUS intervention, and was the intervention manual used in the subsequent REFOCUS trial. The REFOCUS intervention has two components, targeting (a) the patient-worker relationship (called recovery-promoting relationships) and (b) the support offered by the worker (working practices). The REFOCUS intervention is now described.

\section{Component 1: recovery-promoting relationships}

This component comprises several approaches to supporting a partnership-based relationship. Four types of relationships were considered as candidates for use in routine clinical interactions: mentoring, 'real relationships', trialogues and coaching.

Mentoring involves an experienced person (the worker) assisting another (the patient) in developing specific skills and knowledge. Although widely used in the business world, no research using mentoring as a worker interaction style in a mental

\begin{tabular}{|c|c|c|}
\hline Source & Theory & Implication for REFOCUS intervention \\
\hline 1 & $\begin{array}{l}\text { Meaningful involvement of people with lived } \\
\text { experience is needed }\end{array}$ & $\begin{array}{l}\text { People with lived experience are involved as co-applicants, in advisory committees, } \\
\text { as researchers, and in analysis and dissemination }\end{array}$ \\
\hline 1 & Clinical guidelines are indexed on diagnosis & The intervention is transdiagnostic, but evaluation will focus on people with psychosis \\
\hline 2 & Patients value individualised care & Care planning starts with the individual's values and preferences \\
\hline 2 & Recovery is an active process & The intervention focuses on supporting self-management rather than 'fixing the problem' \\
\hline 2 & Recovery is individual and unique & The intervention is flexible and non-prescriptive \\
\hline 3 & $\begin{array}{l}\text { Recovery can occur without professional } \\
\text { intervention }\end{array}$ & $\begin{array}{l}\text { Clinical expertise is offered as a support, and recognising other types of help may also } \\
\text { contribute to recovery }\end{array}$ \\
\hline 3 & $\begin{array}{l}\text { Different support is needed at different stages of } \\
\text { recovery }\end{array}$ & $\begin{array}{l}\text { The target group is people using community-based mental health services, to reduce } \\
\text { heterogeneity }\end{array}$ \\
\hline 3 & $\begin{array}{l}\text { Key recovery processes are Connectedness, } \\
\text { Hope, Identity, Meaning and Empowerment (CHIME) }\end{array}$ & $\begin{array}{l}\text { The intervention content targets these five processes, and CHIME is used to inform the } \\
\text { outcome evaluation strategy }\end{array}$ \\
\hline 4 & $\begin{array}{l}\text { Practical support, diagnosis and medication remain } \\
\text { important }\end{array}$ & The intervention is in addition to, rather than replacing, current mental health practice \\
\hline 4 & Some patients are sceptical about recovery & The term 'recovery' and associated concepts are used only where helpful \\
\hline 5 & Recovery support spans four domains of practice & $\begin{array}{l}\text { The intervention targets the domains relating to front-line practice: supporting personally } \\
\text { defined recovery and working relationships }\end{array}$ \\
\hline 6 & $\begin{array}{l}\text { Team members hold differing understandings } \\
\text { of recovery }\end{array}$ & $\begin{array}{l}\text { The intervention is provided to teams, to support the development of a team-level } \\
\text { understanding of recovery }\end{array}$ \\
\hline
\end{tabular}


health system could be located. (Although there was a report of a pilot involving people with lived experience mentoring psychiatrists, (www.dorsetmentalhealthforum.org.uk/recovery.html.)

A real relationship is one in which 'each is genuine with the other and perceives the other in ways that befit the other. ${ }^{21}$ Although being perceived as a person rather than a patient is reported by some patients as a turning point in their recovery journey, the real relationship concept has emerged from psychotherapy rather than general mental health services, and its usefulness in a context sometimes involving issues of compulsion and capacity is unknown.

A trialogue meeting is a community forum where patients, carers, friends, mental health workers and others with an interest in mental health participate in an open dialogue. In Germanspeaking countries, well over 100 trialogue groups are regularly attended by 5000 people. $^{22}$ However, evaluation is limited and its structure makes it difficult to incorporate into routine clinical work.

Coaching was chosen as the focus for the staff training component of the REFOCUS intervention. Coaching is widely used, has socially acceptable positive connotations relating to strengths (rather than the problem-focused connotations of 'therapy') and has been used in mental health services. For example, the Collaborative Recovery Model uses coaching to support goal-striving by patients. ${ }^{23}$

Recovery-promoting relationships were supported using five approaches. First, staff training using a locally developed Coaching Conversations for Recovery training programme. Second, the grounded theory study of staff perspectives on recovery-oriented practice $^{12}$ found that staff had a range of opinions about recovery, reflecting their need to balance competing priorities and demands placed on them. The development of a shared team understanding was included as a training goal. Third, staff values underpin practice and 'staff role perception' was identified as influential, ${ }^{12}$ so a goal of staff training was to give a safe opportunity to explore values held by individual workers. Fourth, to give an opportunity for a non-role-defined experience of relating to each other (and hence reduce any 'them-and-us' beliefs about having little in common), the undertaking of a Partnership Project was encouraged, in which staff and patients from the same team take on a joint and non-clinical task, with a small amount of resources ( $£ 500$ per team). Fifth, because both parties are active agents in the relationship, the intervention tried to raise expectations in patients about being actively involved in the working relationship, and to encourage them to bring their expertise by experience to inform the clinical discussions.

\section{Component 2: working practices}

Supporting personal recovery involves providing interventions and treatments in the service of the person's recovery, i.e. led by what the individual identifies as needed. Three challenges were identified: planning support based on the individual's values rather than clinical priorities; amplifying strengths as well as ameliorating deficits; and planning care based as much as possible on the goals of the patient. Each led to a specific working practice.

Working Practice 1 is understand values and treatment preferences. Traditional clinical assessment processes can inadvertently reinforce an identity as a patient, ${ }^{24}$ whereas if services are to be oriented around the individual (i.e. patientcentred) then the starting point for assessment needs to be a rich understanding of a person's identity. This involves a strong focus on understanding what matters to the individual (i.e. their values) and what if any support they want from mental health services (i.e. their treatment preferences). Resources supporting Working
Practice 1 comprised a conversational approach using a Values and Treatment Preferences form; a narrative approach supporting the patient to develop their own story; and a visual approach using life mapping.

Working Practice 2 is assessing strengths. It has been proposed that clinical assessment should focus on four dimensions: (a) deficiencies and undermining characteristics of the person; (b) strengths and assets of the person; (c) lacks and destructive factors in the environment; and (d) resource and opportunities in the environment. ${ }^{25}$ Traditional clinical assessment focuses on dimension 1, and there is no doubt that ameliorating intrapsychic deficits, such as reducing symptoms or social disability, is an important contribution to recovery. The REFOCUS intervention seeks to extend clinical expertise to also include dimension 2. Our systematic review of strengths measures ${ }^{13}$ recommended the Strengths Assessment Worksheet (SAW $)^{26}$ as the most widely used and evaluated qualitative measure of strengths. Staff training in using the SAW to inform care planning was included in the intervention. Resources supporting Working Practice 2 were the SAW and strengths assessment techniques.

Working Practice 3 is supporting goal-striving. Consistent with the substantial evidence from research into self-management and shared decision-making, helping people to - with appropriate support - do things for themselves is a central orientation of a recovery-focused mental health service. However, evidence from reviewing care plans indicates that - at least as recorded - actions are primarily undertaken by staff. For example, a review of 3526 care plan action points for 700 patients found 2489 (71\%) were for staff to action, with only 725 (21\%) for joint action and 287 $(8 \%)$ for action by the patient. ${ }^{27}$ Therefore, the third working practice was focused on supporting patients to identify, strive towards and achieve personally valued goals. Resources supporting Working Practice 3 were the GROW model of coaching $^{28}$ to identify and plan actions towards personally valued goals.

Six implementation strategies were developed through advisory committee consultation and Qualitative Study $3:^{12}$ (a) information sharing with staff and patients through letters and meeting to raise expectations; (b) 1.5 days of personal recovery training sessions for staff involving people with lived experience as trainers; (c) 2 days of coaching skills training for staff; (d) five team manager reflection sessions; (e) six team reflections sessions; and (f) reflection in supervision.

\section{Stage 3 (REFOCUS model)}

The REFOCUS model was developed to describe the proposed causal pathway from receiving the intervention to improved recovery, ${ }^{2}$ and is shown in Fig. 1.

Staff practice change is based on the theory of planned behaviour. Team and individual values reflect the behavioural influence of subjective norms. Attitude, knowledge and skill reflect the behavioural influence of behavioural control.

The impact on the experience of the patient occurs in relation to both content (support) and process (relationships) of care. A systematic review of recovery support measures (15738 articles screened, 371 reviewed) identified six measures, none of which could be recommended. ${ }^{16}$ Therefore, a new measure called INSPIRE was developed, which has subscales assessing the value placed on the support received (individualised to reflect the values and treatment preferences of the patient) and the relationship with the worker. $^{29}$

Four proximal outcome domains were identified. Quality of life is a standard patient-rated outcome measure. The CHIME framework of recovery processes informed the choice of three 
other proximal outcome domains: hope, well-being and empowerment. A systematic review of Hope showed the construct to be important, and identified a candidate pool of eight measures from 20150 articles screened and 721 reviewed. ${ }^{14}$ Identity and meaning link with the emerging construct of well-being, ${ }^{30}$ and a systematic review of well-being identified a candidate pool of 20 measures from 19337 articles screened and 421 reviewed. ${ }^{31}$ Finally, the outcome domain of Empowerment is an intended benefit from coaching. No useable measure of Connectedness was identified.

A systematic review showed that the predefined distal outcome of personal recovery was measurable,${ }^{15}$ and identified a candidate pool of 12 measures from 31237 articles screened and 336 reviewed. The questionnaire about the Process of Recovery ${ }^{32,33}$ was recommended for use.

\section{Discussion}

The MRC Framework for Evaluating Complex Interventions was used to develop a testable and empirically defensible pro-recovery intervention. The theory base included existing research synthesised in seven systematic reviews and two narrative reviews, and three qualitative studies addressing key knowledge gaps. The resulting REFOCUS intervention is intended to increase the support for recovery provided by community mental health teams. The intervention is transdiagnostic and transprofessional, so in principle it may have relevance (following modification and evaluation) in other settings, such as in-patient, private practice, peer-run services or other clinical populations.

\section{Strengths and limitations}

The REFOCUS programme was funded for 5 years, allowing 18 months for the intellectual work reported here. This had several advantages. As teams are built not formed, having the time to develop a knowledgeable, reflective and high-performing research team may have improved the intellectual quality of the output. We believe this is more likely to lead to innovation than separate projects over the same length of time. Overall, the intervention is based on a coherent synthesis (and in most cases peer-reviewed publication) of a wide range of evidence. Finally, the timeframe and financial resources permitted the 'higher demands on resources and slower pace of research' (p. 65) ${ }^{34}$ required for meaningful patient public involvement.

The application of the MRC Framework to the development of the intervention was relatively rigorous. However, a recent methodological extension of the framework identifies theory-driven approaches to evaluation. ${ }^{35}$ The extension provides case studies relating to peer counselling for maternal depression, community-based rehabilitation for schizophrenia, and integration of mental health and primary care systems in low- and middleincome countries. Some features recommended in the extension were used in the REFOCUS programme, including a participatory approach, and clarity about causal pathways and intended impact. Others, such as making assumptions about underpinning causal pathways explicit and identifying preconditions for successful implementation, were not, and would have enhanced the design.

Knowledge from implementation science research was inadequately applied in the REFOCUS programme. The resulting limitations include the lack of clarity about the optimal level of challenge to staff practice, the development of implementation strategies with less rigour than the development of the intervention, and the absence of piloting of the intervention.

To make the study manageable, several important aspects were not addressed in the REFOCUS intervention. A main limitation relates to the minimal approach taken to harnessing the resource

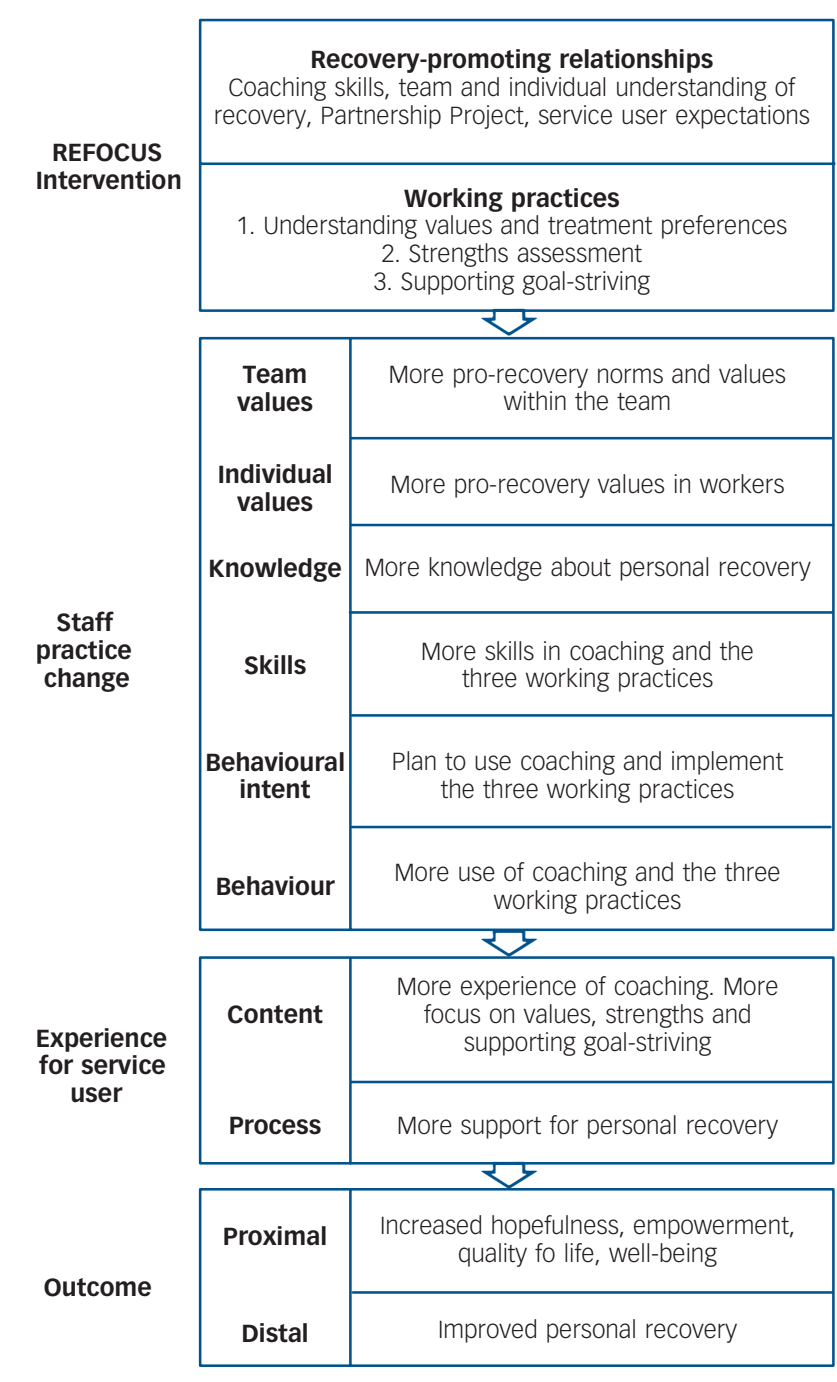

Fig. 1 The REFOCUS model.

of lived experience. ${ }^{36}$ The REFOCUS intervention primarily targets the staff side of the dyadic relationship between worker and patient, with modest efforts made to raise patient expectations through an information session and a letter. A more effective strategy would involve actively targeting both sides of the relationship. Emerging approaches include making 'credible role models of recovery' ${ }^{37}$ more visible by employing peer support workers in services, and supporting active involvement in clinical decision-making.

Second, beyond some involvement in LEAP, the study did not incorporate the perspective of carers. There is only a small and primarily qualitative or survey-based evidence base concerning carer perspectives on recovery. As family and friends are so influential on recovery, this is an important evidence gap.

Third, a decision was made to focus on the two domains from the Recovery Practice Framework relating to direct clinical practice. The REFOCUS intervention was intended to be integrated into existing practice, consistent with an assumption that many current 'clinical recovery' practices, such as evidencebased interventions and social care, directly contribute to the personal recovery of many patients. The goal was therefore not to develop an alternative service system, but rather for new research to inform and amplify the best aspects of current mental health practice. The remaining two domains of the Recovery Practice Framework are also important. The organisational 
commitment domain is being addressed in England through the Implementing Recovery through Organisational Change (ImROC) programme (www.imroc.org). The programme is consistent with REFOCUS in being based on the view that 'If recovery is really going to be the defining feature of our mental health services, there needs to be a fundamental change in the quality of day-to-day interactions' (p. 2). ${ }^{38}$ However, the ImROC approach focuses on organisational transformation. Other national approaches are underway in Australia (www.health. gov.au/internet/main/publishing.nsf/Content/mental-pir) and USA (samhsa.gov/recoverytopractice).

Finally, the promoting citizenship domain - what in the UK is called social inclusion and in USA community integration - was not directly addressed in the intervention or as an outcome in the model. This has been rightly highlighted as a weakness of REFOCUS, ${ }^{39}$ and indeed it has been suggested that 'the largest contribution by mental health services to supporting recovery may come from enabling the empowerment of patients to experience the full entitlements of citizenship' (p. 52). ${ }^{40}$ There is an urgent need for new and evaluated approaches to increasing social cohesion and social capital. ${ }^{41}$

\section{Implications}

The next step for the REFOCUS intervention is formal evaluation. Phase 2 of the REFOCUS programme is a multisite cluster randomised controlled trial (ISRCTN02507940) involving over 400 patients. ${ }^{42,43}$ In England, the REFOCUS intervention is also being evaluated in mental health trusts participating in the Innovation Network following from the Schizophrenia Commission, and the PULSAR Study in Australia is crossculturally modifying the REFOCUS intervention and extending it to primary care settings.

An important knowledge transfer strategy has been active and free dissemination of developed materials. The study website (researchintorecovery.com) contains downloadable versions of the REFOCUS manual, INSPIRE, SAFE and other resources. As a result, the study is making a broader impact on policy and practice. For example, the Recovery Practice Framework underpins the Australian national framework, ${ }^{44}$ and the INSPIRE measure is in use in the ImROC network, recommended for routine use in England, ${ }^{45}$ and being translated into Danish, Estonian, German, Italian, Russian, Slovenian, Spanish and Swedish. Overall, the ambitious goal of providing evidence-based and effective support for people using mental health services to live a life beyond illness may be one step closer.

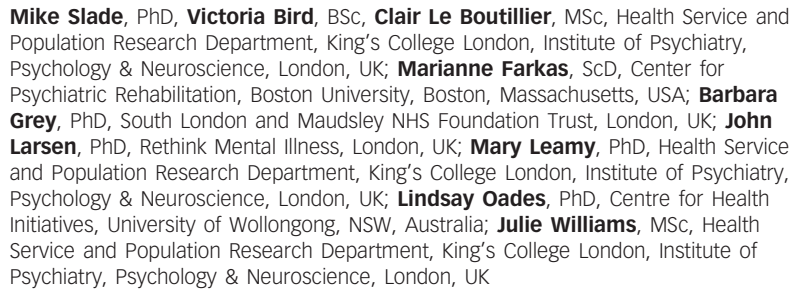

Mike Slade, PhD, Victoria Bird, BSC, Clair Le Boutillier, MSC, Health Service and Population Research Department, King's College London, Institute of Psychiatry, Psychology \& Neuroscience, London, UK; Marianne Farkas, SCD, Center for Psychiatric Rehabilitation, Boston University, Boston, Massachusetts, USA; Barbara Grey, PhD, South London and Maudsley NHS Foundation Trust, London, UK; John Larsen, PhD, Rethink Mental IIIness, London, UK; Mary Leamy, PhD, Health Service and Population Research Department, King's College London, Institute of Psychiatry, Psychology \& Neuroscience, London, UK; Lindsay Oades, PhD, Centre for Health Initiatives, University of Wollongong, NSW, Australia; Julie Williams, MSC, Health Service and Population Research Department, King's College London, Institute of Psychiatry, Psychology \& Neuroscience, London, UK

Correspondence: Mike Slade, PhD, King's College London, Health Service and Population Research Department, Institute of Psychiatry, Psychology \& Neuroscience, London SE5 8AF, UK. Email: mike.slade@kcl.ac.uk

First received 7 Aug 2014, final revision 16 Jan 2015, accepted 13 Feb 2015

\section{Acknowledgements}

We thank the REFOCUS advisory committees for sharing their expertise and the REFOCUS research team at 2gether NHS Foundation Trust for help with data collection. We acknowledge the support of the NIHR Mental Health Research Network.

\section{Funding}

V.B., C.L.B., M.L. and J.W. are funded by a National Institute for Health Research (NIHR) Program Grant for Applied Research. M.S. is supported by the NIHR Biomedical Research Centre for Mental Health at South London and Maudsley NHS Foundation Trust and King's College London. V.B. does consultancy for the National Collaborating Centre for Mental Health. This article presents independent research funded by the National Institute for Health Research (NIHR) under its Programme Grants for Applied Research (PGFAR) Programme (Grant Reference Number RP-PG-0707-10040), and in relation to the NIHR Biomedical Research Centre for Mental Health at South London and Maudsley NHS Foundation Trust and King's College London. The funders did not have a role in the study design, collection, analysis and interpretation of data, in the writing of the report, and in the decision to submit the article for publication. The project will be published in full in the NIHR PGfAR journal. The views expressed in this publication are those of the authors, and the views and opinions expressed by interviewees are those of the interviewees, and do not necessarily reflect those of the NHS, the NIHR, MRC, CCF, NETSCC, the PGfAR programme or the Department of Health. Further information available at researchintorecovery.com/refocus.

\section{References}

1 Leamy M, Clarke E, Le Boutillier C, Bird V, Choudhury R, Macpherson R, et al. A national survey of recovery practice in community mental health teams. $\mathrm{Br}$ $J$ Psychiatry 2015; in press.

2 Bird V, Leamy M, Le Boutillier C, Williams J, Slade M. REFOCUS: Promoting Recovery in Community Mental Health Services. Rethink, 2011.

3 Craig P, Dieppe P, Macintyre S, Michie S, Nazareth I, Petticrew M. Developing and evaluating complex interventions: the new Medical Research Council guidance. BMJ 2008; 337: 979-83.

4 Grant MJ, Booth A. A typology of reviews: an analysis of 14 review types and associated methodologies. Health Info Libr J 2009; 26: 91-108.

5 Mental Health "Recovery" Study Working Group. Mental Health "Recovery": Users and Refusers. Wellesley Institute, 2009.

6 Leamy M, Bird V, Le Boutillier C, Williams J, Slade M. A conceptual framework for personal recovery in mental health: systematic review and narrative synthesis. Br J Psychiatry 2011; 199: 445-52.

7 Slade $M$, Leamy M, Bacon F, Janosik M, Le Boutillier C, Williams J, et al. International differences in understanding recovery: systematic review. Epidemiology Psychiatr Sci 2012; 21: 353-64.

8 Bird V, Leamy M, Tew J, Le Boutillier C, Williams J, Slade M. Fit for purpose? Validation of the conceptual framework of personal recovery with current mental health service users. Aust N Z J Psychiatry 2014; 48: 644-53.

9 Le Boutillier C, Leamy M, Bird VJ, Davidson L, Williams J, Slade M. What does recovery mean in practice? A qualitative analysis of international recoveryoriented practice guidance. Psychiatr Serv 2011; 62: 1470-6.

10 Bird V, Le Boutillier C, Leamy M, Williams J, Bradstreet S, Slade M. Evaluating the feasibility of complex interventions in mental health services: standardised measure and reporting guidelines. Br J Psychiatry 2014; 204: 316-21.

11 van der Krieke L, Bird V, Leamy M, Bacon F, Dunn R, Pesola F, et al. The feasibility of implementing recovery, psychosocial and pharmacological interventions for psychosis: comparison study. Implement Sci 2015; 10: 73.

12 Le Boutillier C, Slade M, Lawrence V, Bird V, Chandler R, Farkas M, et al. Competing priorities: staff perspectives on supporting recovery. Adm Policy Ment Health 2015; 42: 429-38.

13 Bird V, Le Boutillier C, Leamy M, Larsen J, Oades LG, Williams J, et al. Assessing the strengths of mental health service users - systematic review. Psychol Assess 2012; 24: 1024-33.

14 Schrank B, Bird V, Rudnick A, Slade M. Determinants, self-management strategies and interventions for hope in people with mental disorders: systematic search and narrative review. Soc Sci Med 2012; 74: 554-64.

15 Shanks V, Williams J, Leamy M, Bird VJ, Le Boutillier C, Slade M. Measures of personal recovery: a systematic review. Psychiatr Serv 2013; 64: 974-80.

16 Williams J, Leamy M, Bird V, Harding C, Larsen J, Le Boutillier C, et al. Measures of the recovery orientation of mental health services: systematic review. Soc Psychiatry Psychiatr Epidemiol 2012; 47: 1827-35.

17 Tew J, Ramon S, Slade M, Bird V, Melton J, Le Boutillier C. Social factors and recovery from mental health difficulties: a review of the evidence. $\mathrm{Br} J \mathrm{SOC}$ Work 2012; 42: 443-60.

18 Slade M, Bird V, Chandler R, Fox J, Larsen J, Tew J, et al. The contribution of advisory committees and public involvement to large studies: case study. BMC Health Serv Res 2010; 10: 323.

19 Ajzen I. The theory of planned behaviour: reactions and reflections. Psychol Health 2011; 26: 1113-27.

20 McEachan RRC, Conner M, Taylor NJ, Lawton RJ. Prospective prediction of health-related behaviours with the Theory of Planned Behaviour: a meta-analysis. Health Psychol Rev 2011; 5: 97-144. 
21 Gelso CJ, Kelley FA, Fuertes JN, Marmarosh C, Holmes SE, Costa C, et al. Measuring the real relationship in psychotherapy: initial validation of the therapist form. J Couns Psychol 2005; 52: 640-9.

22 Bock T, Priebe S. Psychosis seminars: an unconventional approach. Psychiat Serv 2005; 56: 1441-3.

23 Slade $\mathrm{M}$, Amering $\mathrm{M}$, Oades L. Recovery: an international perspective. Epidemiol Psichiatr Soc 2008; 17: 128-37.

24 Barrett RJ. The Psychiatric Team and the Social Definition of Schizophrenia: An Anthropological Study of Person and Illness. Cambridge University Press 1996.

25 Wright BA, Lopez SJ. Widening the diagnostic focus. A case for including human strengths and environmental resources. In Handbook of Positive Psychology (eds CR Snyder, SJ Lopez): 26-44. Oxford University Press, 2002.

26 Rapp C, Goscha RJ. The Strengths Model: Case Management with People with Psychiatric Disabilities, 2nd edition. Oxford University Press, 2006.

27 Gilburt H, Slade M, Bird V, Oduola S, Craig TK. Promoting recovery-oriented practice in mental health services: a quasi-experimental mixed-methods study. BMC Psychiatry 2013; 13: 167.

28 Alexander G. Behavioural coaching - the GROW model. In Excellence in Coaching (ed. J Passmore): 83-93. Kogan Page, 2006.

29 Williams J, Leamy M, Bird V, Le Boutillier C, Norton S, Pesola F, et al. Development and evaluation of a measure to identify mental health service support for recovery (INSPIRE). Soc Psychiatry Psychiatr Epidemiol 2015; 50: 777-86

30 Schrank B, Riches S, Coggins T, Tylee A, Slade M. From objectivity to subjectivity: conceptualisation and measurement of well-being in mental health. Neuropsychiatry 2013; 3: 525-34.

31 Schrank B, Bird V, Tylee A, Coggins T, Rashid T, Slade M. Conceptualising and measuring the well-being of people with psychosis: systematic review and narrative synthesis. Soc Sci Med 2013; 92: 9-21.

32 Neil S, Kilbride M, Pitt L, Nothard S, Welford M, Sellwood W, et al. The questionnaire about the process of recovery (QPR): a measurement tool developed in collaboration with service users. Psychosis 2009; 1: 145-55.

33 Williams J, Leamy M, Pesola F, Bird V, Le Boutillier C, Slade M. Psychometric evaluation of the Questionnaire about the Process of Recovery (QPR). Br J Psychiatry, 2015; in press.
34 Staley K. Exploring Impact: Public Involvement in NHS, Public Health and Social Care Research. INVOLVE, 2009.

35 De Silva M, Breuer E, Lee L, Asher L, Chowdhary N, Lund C, et al. Theory of change: a theory-driven approach to enhance the Medical Research Council's framework for complex interventions. Trials 2014; 15: 267.

36 Slade $M$, Amering $M$, Farkas M, Hamilton B, O'Hagan M, Panther $G$, et al. Uses and abuses of recovery: implementing recovery-oriented practices in mental health systems. World Psychiatry 2014; 13: 12-20.

37 Davidson L, Bellamy C, Guy K, Miller R. Peer support among persons with severe mental illnesses: a review of evidence and experience. World Psychiatry 2012; 11: 123-8

38 Sainsbury Centre for Mental Health. Implementing Recovery. A New Framework for Organisational Change. Sainsbury Centre for Mental Health 2009.

39 Shera W, Ramon, S. Challenges in the implementation of recovery-oriented mental health policies and services. Int J Ment Health 2013; 42: 17-42.

40 Slade M, Leamy M, Bird V, Le Boutillier C. Mental health services and recovery. In Improving Mental Health Care: The Global Challenge (eds G Thornicroft, M Ruggeri, D Goldberg): 40-56. John Wiley, 2013.

41 Priebe S, Burns T, Craig TK. The future of academic psychiatry may be social. Br J Psychiatry 2013; 202: 319-20.

42 Slade M, Bird V, Le Boutillier C, Williams J, McCrone P, Leamy M. REFOCUS trial: protocol for a cluster randomised controlled trial of a pro-recovery intervention within community based mental health teams. BMC Psychiatry 2011; 11: 185

43 Slade M, Bird V, Clarke E, Le Boutillier C, Mccrone P, Macpherson R, et al. Supporting recovery in patients wih psychosis using adult mental health teams (REFOCUS): a multi-site cluster randomised controlled trial. Lancet Psychiatry 2015; 2: 503-14.

44 Commonwealth of Australia. A National Framework for Recovery-oriented Mental Health Services. Policy and Theory. Australian Health Ministers' Advisory Council, 2013.

45 Shepherd G, Boardman J, Rinaldi M, Roberts G. ImROC Briefing Paper 8. Supporting Recovery in Mental Health Services: Quality and Outcomes. Implementing Recovery through Organisational Change, 2014. 